\title{
Effect of Early Amino Acids Supplementation on Serum Glucose and Calcium of Very Low Birth Weight Premature Infants
}

\author{
Ghassan Saleh Ahmed Salama* and Yasser Fouad Mahmoud Amer \\ Paediatric Department, Neonatologist, Qurayyat General Hospital, Saudi Arabia
}

*Corresponding author: Ghassan Saleh Ahmed Salama, Paediatric Department, Neonatologist, Qurayyat General Hospital, Saudi Arabia

Received: 罡January 28, 2019

Published: 䟧February 05, 2019

\begin{abstract}
Objective: To examine effects of early amino acid (AA) infusion on serum glucose and calcium levels of very low birth weight (VLBW) preterm newborns, through the first 72 hours of life.

Study Design: hospital designed prospective study of total 110 VLBW newborns with $<32$ weeks gestation, and less than 1500 g was performed. 55 newborns received AA within 6 hours of life (early group) while 55 newborns did not receive AA during the first 72 hours of life (late group).

Results: Average glucose levels were significantly lower in the early group during the first 72 hours of life. Average serum Ca levels during first 72 hours of life were significantly higher in newborns with early group.

Conclusions: Early amino acid infusion improve serum glucose and Ca levels in VLBW newborns, and thus decreasing the risk of hyperglycemia and hypocalcemia among VLBW infants.
\end{abstract}

Keywords: Amino acids (AA); Glucose; Calcium

\section{Introduction}

Very Low birth weight infants (VLBW) are born at a time when the fetus is undergoing rapid intrauterine brain and body growth Continuation of this growth in the first several weeks postnatally is often a challenge because these infants are usually highly stressed and at risk for catabolism. [1] Parenteral nutrition is needed in these infants because most cannot meet the majority of their nutritional needs using the enteral route. Despite adoption of a more aggressive approach with amino acid infusions by the AAP, there still appears to be a reluctance to use early intravenous amino acid infusions in many N ICUs around the world. Approximately $40 \%$ of premature neonates $<1,250 \mathrm{~g}$ develop hyperglycemia defined as serum glucose $>125 \mathrm{mg} / \mathrm{dl}$ with glucose loads as low as 4-6 mg/ $\mathrm{kg} / \mathrm{min} 2$ [2-4]. This hyperglycemia is thought to be a result of the inability to inhibit gluconeogenesis in response to a continuous glucose infusion, absolute or relative insulin insufficiency followed by hepatic and peripheral insulin resistance [5]. The lack of nutritional reserves result in protein imbalance within 6 hours after birth [6,7]. and the delay in commencing total parentaral nutrition (TPN) can cause early muscle protein breakdown [8] Higher concentrations of protein in the diet have shown to improve the carbohydrate metabolism and utilization in VLBW newborns $[9,10]$. There are no reports of changes in early versus late amino acid infusion on glucose infusion rates and pH levels in VLBW newborns to our knowledge. Calcium is playing a key role in many cellular and extracellular biochemical processes. VLBW newborns are at risk of a significant decrease in serum calcium levels within first 48 hours of life as compared to term newborns because of a delayed response of parathyroid and calcitonin hormones [11,12]. Few studies concentrated on hypercalcemia as a complication of amino acids introduction to VLBW newborns $[13,14]$. but, to our knowledge no single report compared the effect of early versus late 
introduction of amino acids on serum calcium of VLBW newborns. The objective of this study was to determine whether early amino acid administration in the VLBW premature neonate would decrease the incidence of hyperglycemia within first 72 hours of life. We determined the effect of amino acids supplementation on serum calcium levels of VLBW newborns. In addition, we compared average $\mathrm{pH}$ levels, in the presence of early versus late amino acids administration.

\section{Subjects and Methods}

This was a hospital designed prospective study conducted after obtained consents from the included infants parents and approval from the research ethical committee of the Qurayyat General Hospital / KSA between 2017-2018. A convenience sample size of 110 newborns born at less than 32 weeks gestation and less than $1500 \mathrm{~g}$ who survived beyond 72 hours of life was included. Infants were divided randomly using the odd and equal numbers of the patient admission sequence in two groups: Early group ( $\mathrm{N}=55)$, supplemented with 2 grams $/ \mathrm{kg} /$ day of $10 \%$ amino acids within less than 6 hours from birth. And with two variations of dextrose concentration (dextrose 5\% for preterm infants who are less than 1000 grams at birth and $10 \%$ for those who are more than 1000 grams at birth). Late group ( $\mathrm{N}=55)$, started TPN including amino acids more than 72 hours from birth, and infused only with two variations of dextrose concentration (dextrose 5\% for preterm infants who are less than 1000 grams at birth and 10\% for those who are more than 1000 grams at birth) during the first 72 hours after birth. Newborns that had major congenital abnormality or malformations were excluded from the study. Crossover was not allowed between groups. All blood samples withdrawn by

\section{Results}

Table 1: Patient characteristics.

\begin{tabular}{|c|c|c|c|}
\hline \multicolumn{4}{|c|}{ Patients $(\mathrm{N}=110)$} \\
\hline Parameters & Early AA and Ca Group (N =5) & Late AA and Ca Group $(\mathrm{N}=55)$ & $P$ value \\
\hline Gestational age (Week) & $29.87 \pm 2.75$ & $29.17 \pm 2.53$ & 0.886 \\
\hline Birth weight (grams) & $1057 \pm 265$ & $1011 \pm 290$ & 0.252 \\
\hline Apgar Scores at $5 \mathrm{~min}$ & $7.25 \pm 1.9$ & $7.31 \pm 1.5$ & 0.51 \\
\hline Maternal chorioamnionitis & $21(38 \%)$ & $18(33 \%)$ & 0.662 \\
\hline $\mathrm{PROM} *$ & $15(27 \%)$ & $9(16 \%)$ & 0.254 \\
\hline Male gender & $30(55 \%)$ & $23(42 \%)$ & 0.646 \\
\hline Antenatal steroids & $30(73 \%)$ & $24(75 \%)$ & 0.814 \\
\hline Start time of TPN (hour of life) & $2.2 \pm 1.4$ & $71.4 \pm 1.9$ & $<0.0001$ \\
\hline
\end{tabular}

*PROM= Premature Rupture of Membranes.

The two groups were comparable for demographic characteristics, with no statistical significance for any parameter except the time to initiation of amino acids (Table 1). There were no statistical differences between the early and late groups regarding birth weight and gestational age, distribution of gender, a registered nurse and sent to the local laboratory at Qurayyat General Hospital / KSA. All enrolled infants were rounded and examined on a daily base by the neonatologist. Data about baseline maternal and neonatal demographics, composition of continuous infusions, serum glucose, serum calcium, serum albumin, and blood gases were collected during the first 72 hours of life. The time span of 72 hours was determined because many NICUs start the TPN only after the 72 hours of life. While, in our unit most of the newborns receive TPN within 24 hours of birth. We expected the effects on early TPN versus late TPN would be balanced by 72 hours of life. Hyperglycemia was defined as serum glucose levels of $>125 \mathrm{mg} / \mathrm{dl}$.

Hypoglycemia was defined as serum glucose levels of $<40 \mathrm{mg} /$ dl. Hypocalcemia was defined as serum calcium levels less than $7 \mathrm{mg} / \mathrm{dl}$. Intravenous Ca-gluconate boluses were given if calcium between $7-6.5 \mathrm{mg} / \mathrm{dl}$ but, the infant is asymptomatic, while the infant maintained on Intravenous Ca-gluconate at any calcium level if the infant symptomatic, or if calcium $\leq 6.5$ even asymptomatic. Comparison of glucose infusion rates and average glucose levels for each day of life across the amino acids groups was a primary outcome measure. Differences between calcium levels, and acidosis across the groups were secondary outcome variables. Statistical analysis was conducted using SPSS statistical package (version 18). Baseline perinatal variables were compared between the early group and late group. Differences between average glucose levels, calcium levels, and pH levels were compared between the early group and late group using the unpaired t-test. Need for calcium boluses were compared using the chi-squared test. No corrections for multiple comparisons were made. The level of significance was set at $\mathrm{P}<0.05$. 
as compared to the late group. The level of significance was higher during first 24 hours $(\mathrm{P}=0.02)$ as compared to 48 hours $(\mathrm{P}=0.047)$. Average glucose levels were not significant between 48 to 72 hours of life between the groups. Glucose infusion rates were not different between the early and late groups during the first 3 days of life. Calcium boluses were required by $17.5 \%$ of newborns in the early group vs. $28 \%$ in the late group. This difference was not statistically significantly significant $(\mathrm{P}=0.272)$ (Table 2$)$. But this was clinically significant as 3 of the newborns in the late group developed symptomatic hypocalcemia in form of convulsions and needed to be maintained on intravenous calcium gluconate. Average $\mathrm{pH}$ levels trended higher in early group (Table 3). The pH levels were significantly higher $(\mathrm{P}=0.04)$ during second day in the early group $(7.32 \pm 0.01)$ as compared to the late group (7.26 \pm 0.02$)$.

Table 2: Glucose levels (mg/dl) and glucose infusion rates (GIR) (mg/kg/min).

\begin{tabular}{|c|c|c|c|c|c|c|}
\hline \multicolumn{7}{|c|}{ Glucose levels (mg/d) Glucose infusion rates (GIR) (mg/kg/min) } \\
\hline Timeline & Early Group & Late Group & P value & Early Group & Late Group & P value \\
\hline & Glucose & Glucose & & GIR & GIR & \\
\hline Baseline & $58 \pm 3.5$ & $82 \pm 9$ & 0.008 & & & \\
\hline 24 hours & $85 \pm 3.2$ & $99 \pm 4.8$ & 0.02 & $4.6 \pm 0.26$ & $4.1 \pm 0.29$ & 0.16 \\
\hline 48 hours & $102 \pm 3.2$ & $115 \pm 6.1$ & 0.047 & $5 \pm 0.29$ & $4.3 \pm 0.31$ & 0.1 \\
\hline 72 hours & $105 \pm 5$ & $105 \pm 6$ & 0.97 & $5.7 \pm 0.31$ & $5.1 \pm 0.37$ & 0.21 \\
\hline
\end{tabular}

* Glucose levels and glucose infusion rates (GIR) represented as (Mean \pm SEM).

Table 3: Calcium levels, and pH levels during first 3 days of life.

\begin{tabular}{|c|c|c|c|}
\hline \multicolumn{4}{|c|}{ Calcium Levels (mg/dl) } \\
\hline Timeline & Early Group & Late Group & P value \\
\hline 24 hours & $8.4 \pm 0.29$ & $7.4 \pm 0.12$ & 0.003 \\
\hline 48 hours & $8.8 \pm 0.29$ & $8 \pm 0.18$ & 0.01 \\
\hline 72 hours & $9.5 \pm 0.15$ & $9 \pm 0.16$ & 0.021 \\
\hline \multicolumn{4}{|c|}{ pH levels } \\
\hline Timeline & Early Group & Late Group & P value \\
\hline 24 hours & $7.30 \pm 0.016$ & $7.28 \pm 0.015$ & 0.53 \\
\hline 48 hours & $7.32 \pm 0.01$ & $7.26 \pm 0.02$ & 0.04 \\
\hline 72 hours & $7.31 \pm 0.012$ & $7.29 \pm 0.012$ & 0.1 \\
\hline
\end{tabular}

* Values represented as Mean \pm SEM.

\section{Discussion}

This study demonstrates the use of early and modest doses of protein intake in VLBW newborns and its influence on glucose levels and infusion rates and serum calcium levels. Other studies have demonstrated a positive effect on the usage of amino acid solutions on protein metabolism [15-19]. Only a few studies have specifically looked at effects of early amino acids supplementation on glucose infusion rates and incidence of hyperglycemia at different starting timelines of amino acid-containing solutions. Van Den Akker et al. [6] demonstrated that VLBW newborns who immediately received glucose and $2.4 \mathrm{~g} / \mathrm{kg} / \mathrm{d}$ of amino acid infusion postnatally did not have significantly lower glucose levels as compared to those who received glucose infusions only [20]. This study reported glucose levels at the end of second day only, with a trend of higher glucose levels (6.1 vs.4.6 mmol/L) in the late TPN group as compared to early TPN group, but the difference was not statistically significant. We demonstrated a significant reduction in the average glucose levels in the early group during first 24 hours of life and at 48 hours of life as compared to the late TPN group, with comparable glucose infusion rates across the two groups. Radmacher et al. [21] focused on protein metabolism and reported observations similar to our study. Valentine et al. [22] made similar observations through their review article on the use of TPN in VLBW newborns.

Unique strength of our study is that it for the first time describes lower glucose levels in presence of equivalent GIR in VLBW newborns who received early amino acid infusion. Hyperglycemia in the early newborn period is recognized to result in detrimental life-altering sequelae. Alexandrou et al. [23] have demonstrated that VLBW newborns who had hyperglycemia were at a higher risk of mortality and poor developmental outcome. Early infusion of amino acid can improve glucose levels at higher glucose infusion rates. This improved glucose metabolism might also help to reduce morbidities and mortalities associated with hyperglycemia in VLBW newborns including death, intraventricular hemorrhage, retinopathy of prematurity, necrotizing enterocolitis and late-onset sepsis. In addition, this study looked at effect of calcium infusion 
on calcium metabolism. Our study did demonstrate higher calcium levels in newborns when amino acids were infused within 6 hours of life. However, many newborns still had hypocalcemia and needed bolus doses of calcium gluconate. Few studies concentrated on hypercalcemia as a complication of amino acids introduction to VLBW newborns $[13,14]$ but, in our study we did not observe any case of hypercalcemia and that might be because we did not routinely supply the newborns infants with calcium. It was thought, that amino acid infusion associated with decrease of bicarbonate level and a kind of metabolic acidosis but, our study showed that early infusion of amino acids associated with more physiological $\mathrm{pH}$, compared to no amino acid infusion. The single limitation of this study is the small sample size.

\section{Conclusion}

Early amino acid infusion improve serum glucose and Ca levels in VLBW newborns, and thus decreasing the risk of hyperglycemia and hypocalcemia among VLBW infants. A further prospective evaluation still needed.

\section{References}

1. Salama GS, Kaabneh MA, Almasaeed MN, Alquran Mla (2015) Intravenous lipids for preterm infants: a review. Clin Med Insight Pediatr 9: 25-36.

2. Blanco CL, Baillargeon JG, Morrison RL, Gong AK (2006) Hyperglycemia in extremely low birth weight infants in a predominantly Hispanic population and related morbidities. J Perinatol 26(12): 737-741.

3. Hays SP, Smith EO, Sunehag AL (2006) Hyperglycemia is a risk factor for early death and morbidity in extremely low birth-weight infants. Pediatrics 118(5): 1811-1818.

4. Kao LS, Morris BH, Lally KP, Stewart CD, Huseby V, et al. (2006) Hyperglycemia and morbidity and mortality in extremely low birth weight infants. J Perinatol 26(12): 730-736.

5. Denne SC (2007) Regulation of proteolysis and optimal protein accretion in extremely premature newborns. Am J Clin Nutr 85(2): 621S-624S.

6. Te Braake FW, van den Akker CH, Wattimena DJ, Huijmans JG, van Goudoever JB (2005) Amino acid administration to premature infants directly after birth. J Pediatr 147(4): 457-461.

7. Yeung MY (2006) Glucose intolerance and insulin resistance in extremely premature newborns, and implications for nutritional management. Acta Paediatr 95(12): 1540-1547.

8. Heird WC, Kashyap S, Gomez MR (1991) Protein intake and energy requirements of the infant. Semin Perinatol 15(6): 438-448.
9. Rivera A Jr, Bell EF, Bier DM (1993) Effect of intravenous amino acids on protein metabolism of preterm infants during the first three days of life. Pediatr Res 33(2): 106-111.

10. Embleton ND, Cooke RJ (2005) Protein requirements in preterm infants: effect of different levels of protein intake on growth and body composition. Pediatr Res 58(5): 855-860.

11. Aggarwal R, Upadhyay M, Deorari AK, Paul VK (2001) Hypocalcemia in the newborn. Indian J Pediatr 68(10): 973-975.

12. Hillman LS, Rojanasathit S, Slatopolsky E, Haddad JG (1977) Serial measurements of serum calcium, magnesium, parathyroid hormone, calcitonin, and 25-hydroxy-vitamin D in premature and term infants during the first week of life. Pediatr Res 11(6): 739-744.

13. Bonsante F, Iacobelli S, Latorre G, Rigo J, De Felice C, et al. (2013) Initial Amino Acid Intake Influences Phosphorus and Calcium Homeostasis in Preterm Infants - It Is Time to Change the Composition of the Early Parenteral Nutrition. PLoS ONE 8(8): e72880.

14. Gilligan JE, Worthley LI, Way M, Need AG, Dollman WB (1982) Hypercalcemia associated with parenteral amino acid and dextrose infusion. The American Journal of Clinical Nutrition 35(5): 993-996.

15. Rivera A Jr, Bell EF, Bier DM (1993) Effect of intravenous amino acids on protein metabolism of preterm infants during the first three days of life. Pediatr Res 33(2): 106-111.

16. Kadrofske MM, Parimi PS, Gruca LL, Kalhan SC (2006) Effect of intravenous amino acids on glutamine and protein kinetics in low-birthweight preterm infants during the immediate neonatal period. Am J Physiol Endocrinol Metab 290(4): E622-630.

17. Van Goudoever JB, Colen T, Wattimena JL, et al. (1995) Immediate commencement of amino acid supplementation in preterm infants: effect on serum amino acid concentrations and protein kinetics on the first day of life. J Pediatr 127(3): 458-465.

18. Battista MA, Price PT, Kalhan SC (1996) Effect of parenteral amino acids on leucine and urea kinetics in preterm infants. J Pediatr 128(1): 130134.

19. Parimi PS, Kadrofske MM, Gruca LL, Hanson RW, Kalhan SC (2005) Amino acids, glutamine, and protein metabolism in very low birth weight infants. Pediatr Res 58(6): 1259-1264.

20. van den Akker CH, te Braake FW, Wattimena DJ, Voortman G, Schierbeek $\mathrm{H}$, et al. (2006) Effects of early amino acid administration on leucine and glucose kinetics in premature infants. Pediatr Res 59(5): 732-735.

21. Radmacher PG, Lewis SL, Adamkin DH (2009) Early amino acids and the metabolic response of ELBW infants (p1000 g) in three time periods. Journal of Perinatology 29(6): 433-437.

22. Valentine CJ, Puthoff TD (2007) Enhancing parenteral nutrition therapy for the neonate. Nutr Clin Pract 22(2): 183-193.

23. Alexandrou G, Skiold B, Karlen J, Tessma MK, Norman M, et al. (2010) Early hyperglycemia is a risk factor for death and white matter reduction in preterm infants. Pediatrics 25(3): e584-591.

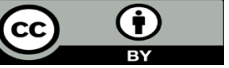

This work is licensed under Creative Commons Attribution 4.0 License

To Submit Your Article Click Here:

Submit Article

\section{PAPN}

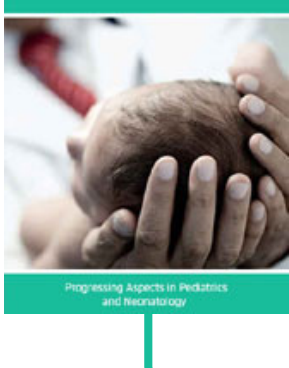

Progressing Aspects in Pediatrics and Neonatology

\section{Assets of Publishing with us}

- Global archiving of articles

- Immediate, unrestricted online access

- Rigorous Peer Review Process

- Authors Retain Copyrights

- Unique DOI for all articles 